\title{
Reversal Atrial Electrical Remodeling Following Cardioversion of Long-Standing Lone Atrial Fibrillation
}

\author{
Eduardo Correa Barbosa, Paulo Roberto Benchimol-Barbosa, Alfredo de Souza Bomfim, Plínio José da Rocha, \\ Silvia Helena Cardoso Boghossian, Denilson Campos de Albuquerque \\ Departamento de Cardiologia - Seção de Eletrofisiologia Cardíaca e Unidade de Arritmia - Hospital Universitário Pedro Ernesto - Universidade \\ Estadual do Rio de Janeiro, Rio de Janeiro, RJ - Brazil
}

\section{Summary}

Background: Atrial fibrillation (AF) itself promotes electrophysiological changes, termed "electrical remodeling", facilitating its recurrence and maintenance. There is evidence that the remodeling process is reversible after restoration of the sinus rhythm (SR). However, the timing for the recovery of electrophysiological properties is still undefined.

Objctive: The aim of this study was to assess the atrial electrical activation using P-wave signal-averaged electrocardiogram (P-SAECG) post-cardioversion of long-standing AF, focusing on the reversal remodeling process to identify the timing of the process stabilization.

Methods: Subjects with lone persistent AF, eligible for cardioversion and successfully converted to SR, were enrolled at the study. SAECG was performed immediately after reversion to SR and repeated on days seven and thirty.

Results: Of 31 subjects, nine presented early recurrence of atrial fibrillation, all of them in the first seven days postcardioversion; 22 remained in SR for at last one month and SAECG was obtained on days seven and thirty after cardioversion. In the latter, P-wave duration progressively abated from the first to the third SAECG (P-wave duration: $185.5 \pm 41.9 \mathrm{~ms}$ vs $171.7 \pm 40.5 \mathrm{~ms}$ vs $156.7 \pm 34.9 \mathrm{~ms}$, respectively, first, second and third SAECG; $p<0.001$ for all matches). In the frequency domain analysis, spectral turbulence was not apparent in SAECG immediately postcardioversion, but sharply increased on day seven and remained unchanged on day thirty.

Conclusion: This study suggests that the first seven days post-cardioversion of long standing AF are critical for reversal remodeling process and arrhythmia recurrence. (Arq Bras Cardiol 2009; 93(2):XXX-XXX)

Key words: Electrocardiography; arrhythmias, cardiac; atrial fibrillation; eletric countershock.

\section{Introduction}

Atrial fibrillation (AF) has been described as a selfperpetuating arrhythmia, which promotes electrophysiological changes in atrial tissue, facilitating its recurrence and maintenance ${ }^{1-5}$. Persistent AF usually progresses to a permanent form and the successful restoration and maintenance of sinus rhythm largely depend on arrhythmia duration, with longstanding AF being more resistant to cardioversion and more prone to recurrence ${ }^{6-9}$.

Both resistance to cardioversion and tendency of recurrence are strongly related to the development of electrophysiological substrates characterized by shortened atrial effective refractory period $^{2,4,5,10-13}$, slow conduction in the atrial myocardium ${ }^{10,11,14-}$ ${ }^{16}$ and dispersion of refractoriness ${ }^{4,13,17,18}$. These changes can be induced by both rapid atrial pacing and AF itself, and have been termed "electrical remodeling" 2 . The resultant electrophysiological abnormality is the presence of multiple wavelets around the atria,

Mailing address: Eduardo Correa Barbosa •

Rua Alzira Cortes, 5/101 - Botafogo - 22.260-050 - Rio de Janeiro, RJ - Brazil. Email: barbosa.md@hotmail.com

Manuscript received June 16, 2008; revised manuscript received July 14, 2008; accepted July 17, 2008. some of which circle back on them creating multiples reentry circuits, as proposed by Moe et al ${ }^{19,20}$ in 1962 and 1964, and later confirmed by Allessie et a ${ }^{21}$ in 1985 .

In animal models, the remodeling process has been demonstrated to be reversible after restoration of the sinus rhythm, and is characterized by progressive increase in both atrial conduction velocity and refractoriness (reversal remodeling) $)^{1,2,10,11,13,22}$. Recovery has also been demonstrated in human atria ${ }^{3,23-26}$. On the other hand, a remarkable vulnerability to recurrence during post-cardioversion period has been observed while reverse remodeling is yet incomplete ${ }^{23}$. However, the necessary time to completely restore atrial electrophysiological properties, especially the conduction velocity, is yet to be defined.

The methods employed for electrophysiological analysis are usually invasive ${ }^{23-25,27}$ and make consecutive analysis of the same subject difficult, generating limitations to determine the moment of complete reversal remodeling. Furthermore, little is known about the effects of antiarrhythmic drugs on the remodeling process, especially amiodarone, frequently used in order to facilitate cardioversion and maintain sinus rhythm ${ }^{28}$. The aim of this study was to assess the atrial electrical 
activation in post-cardioversion of subjects with long-standing $\mathrm{AF}$ to describe the timing of reversal remodeling.

\section{Methods}

\section{Study population}

Subjects with lone persistent atrial fibrillation of $>2$ months duration eligible for cardioversion were included in this study. Lone AF was defined on basis of history (to exclude holiday heart syndrome), physical examination, conventional electrocardiography, chest X-ray, echocardiography, stress testing (when appropriate) and thyroid function tests. Planned exclusion criteria for the study were contraindication to anticoagulation or amiodarone, pregnancy, left atria $\geq 5.5 \mathrm{~cm}$ or age $>80$ years. Additional exclusion criteria were prior use of beta-adrenergic blockers, ACE inhibitors, angiotensin receptor blockers and calcium antagonists. The subjects gave their written informed consent and the local Ethics Committee approved the study protocol.

\section{Study protocol}

All subjects received therapeutic anticoagulation with warfarin before cardioversion with international normalized ratio between 2.0 and 4.0 during three consecutive weeks. Thereafter, oral amiodarone, in daily doses of up to $800 \mathrm{mg}$, was initiated. If sinus rhythm was not obtained in the following 7 days, direct current electrical cardioversion was performed. If sinus rhythm was restored, a daily dose of $200 \mathrm{mg}$ of amiodarone was maintained for one month. The cardioversion protocol consisted of: 1) general IV anesthesia with propofol $2 \mathrm{mg} / \mathrm{kg}$; 2) synchronized DC monophasic sinusoidal waveform shock with anterior-apex paddle placement; 3) Starting energy delivery with 200J, ranging until 360J, if necessary.

\section{P-wave high-resolution electrocardiogram}

P-wave signal-averaged electrocardiogram (P-SAECG) was performed immediately after reversion to sinus rhythm (first P-SAECG) and repeated on the seventh (second P-SAECG) and at the thirtieth (third P-SAECG) day after successful cardioversion. P-SAECG was recorded with a Predictor Ilc System (ART Corazonix, Austin) applying a modified orthogonal montage of three bipolar leads. The $X$ lead was standardized on the $2^{\text {nd }}$ intercostal space at the right sternal border and on the left lower rib border at the hemiclavicular line to present larger and taller P-waves, as previously described. The $Y$ lead was thus placed on $5^{\text {th }}$ intercostal space at the left and the right mid axillary lines and the $Z$ lead placed at the level of $4^{\text {th }}$ intercostal space to left sternal border and its projection in the back. Positive reference electrodes were placed inferior, left and anterior, respectively to the leads $X, Y$ and $Z$. The sampling frequency was set at $2.0 \mathrm{kHz}$. The fiducial point was shifted to the right and the P-wave and PR segment were exposed into the averaging window. Averaging noise was assessed within a $50 \mathrm{~ms}$ window placed on the T-P segment. The averaging was conducted using an R-triggered technique with a correlation window of $40 \mathrm{~ms}$ placed on the ascending limb of P-wave and a correlation coefficient of 0.99 . The threshold for the maximum final averaged noise estimate was set at $0.3 \mu \mathrm{V}$. One trained observer analyzed SAECG in all subjects.

Time domain and spectral turbulence analysis: After signal averaging, the onset and offset points of the analytic region were derived from time domain analysis of the P-wave vector magnitude, represented by the root-sumsquare of independently filtered XYZ-leads, using a least square filter with $100 \mathrm{~ms}$ fitting window. The limiting points of the P-wave vector magnitude was determined by visual inspection, allowing identification of the P-wave duration and determination of the analytic region of the X-lead.

The analytic region was pre-processed to extract the first derivative and thus submitted to time-frequency mapping by short-time Fourier transform. Each data segment was limited in $16 \mathrm{~ms}$, with $2 \mathrm{~ms}$ interval between successive segments to assure adequate time-resolution, tapered by a BlackmannHarris window after mean removal, and zero-padded to 512 points. The boundaries of the analytic region (up to $200 \mathrm{~ms}$ ) were placed $16 \mathrm{~ms}$ prior to the onset of the P-wave and on a point onto the PR segment.

Spectral turbulence was analyzed using 4 parameters previously reported in the literature: the mean (MEC) and the standard deviation (SEC) of the inter-segment spectral correlation and the mean (MET) and the standard deviation (SET) of the signal frequency edge track. The correlation between successive power-spectral estimations was calculated in the range from 0 to $300 \mathrm{~Hz}$. It was studied because an absolutely uniform conduction of the electrical signal during atrial activation is expected to give perfect correlation, while the presence of high frequency components from fragmented conduction will be reflected by decay in correlation mean and increased standard deviation.

To prevent low values of correlation due to the absence of depolarization signal and/or the presence of band-limited white noise from interfering with the analysis, the ratio between the areas of low $(0-30 \mathrm{~Hz})$ and high $(>30 \mathrm{~Hz}-100 \mathrm{~Hz})$ frequencies was employed to verify whether the region was signal or noise. Noise contamination causes spectral energy content distribution to be even between low and high frequency areas. A noisy segment was defined when the value of the noise (low-high) ratio did not reach a threshold. Due to the low energy content of the P-wave, an optimal compromise between noise overestimation and energy content assessment was achieved when noise ratio was set at 30. When the threshold was not reached, including the segments before and after the P-wave, the correlation was set at 1 . The time-series generated by successive correlation values along the analytic region is thus used to extract MEC and SEC.

The frequency edge track was set to detect the frequency that limits the energy of each power-spectral estimation to $80 \%$ of the total. The parameters MET and SET correspond to the mean and the standard deviation of the time series of edge frequencies and are expressed in $\mathrm{Hz}$.

It was assumed that the presence of intense fragmented electrical activity in atria would result in increased values of SEC, MET and SET and decreased values of MEC. 


\section{Statistical analysis}

Continuous variables were expressed as mean \pm SD and compared at follow-up segments using paired or unpaired Student-t test when appropriate. Normality of the estimated probability density function of the variables was assessed by standard skewness tests to validate the tests for mean comparisons. All variables demonstrated appropriate adjustment to normal distribution. MEC and SEC were multiplied by 100 before analysis. The correlation of the variables between follow-up segments was calculated by Pearson's coefficient and tested using ANOVA applied to the correlation. Discrete variables were reported as ratio or percentage and analyzed by either Chi-square or Fisher's exact test, when appropriate. $P$ values $<0.05$ were considered significant.

\section{Results}

In a cohort of 581 consecutive subjects, with all forms of atrial fibrillation, scheduled for treatment at the Arrhythmia Control Unit of University Hospital (Pedro Ernesto University Memorial Hospital, Rio de Janeiro, Brazil) between March 2001 and April 2006, 37 subjects with long-standing lone atrial fibrillation ( $7 \pm 5$ months; ranging from 2 to 24 months) and according to the planned exclusion criteria were eligible for the study. Six subjects were later excluded because cardioversion was ineffective for the restoration of sinus rhythm. Thirtyone subjects, 16 men, mean age of $57 \pm 12$ years, were successfully converted to sinus rhythm and were enrolled. One subject had chemical conversion five days after starting oral amiodarone and thirty had electrical conversion (262.6 \pm 64.7 Joules). The P-SAECG was attempted immediately after cardioversion in all subjects, but one. In this subject, who underwent chemical conversion, P-SAECG was performed within two days after sinus rhythm restoration. Nine subjects underwent early recurrence of atrial fibrillation (Group-I), all of them in the first seven days post-cardioversion. Twentytwo (Group-II) subjects remained in sinus rhythm by at least one month, and P-SAECG was obtained on days seven and thirty after cardioversion. No episodes of thromboembolism were observed during one month of follow-up. Table 1 shows demographic and clinical variables of the subjects.

No significant differences were observed regarding gender, age, left atrial size or ejection fraction between Groups I and II. Subjects from Group-I required significant higher energy output for appropriate arrhythmia conversion than those from Group-II (304.4 \pm 65.4 Joule vs $240.0 \pm 50.3$ Joule; $\mathrm{p}=0.006)$ and presented longer AF duration $(9.9 \pm 6.4$ months vs $4.9 \pm 2.8$ months; $\mathrm{p}=0.02$ ).

\section{P-SAECG analysis}

Figure 1 shows the parameters of P-wave analyzed in the first (Groups I and II), second and third P-SAECG.

The parameters of first P-SAECG were not different between Groups I and II (Figure 1; $p=$ NS). In Group-II, the P-wave duration significantly decreased from the first to the third P-SAECG. This was observed in filtered Vector Magnitude P-wave duration (VM P-wave duration: 185.5 $\pm 41.9 \mathrm{~ms}$; $171.7 \pm 40.5 \mathrm{~ms}$ and $156.7 \pm 34.9 \mathrm{~ms}$, for respectively first, second and third SAECG; $p<0.001$ for all matches). At the
Table 1 - Clinical Characteristics of the subjects in groups I and II *:

\begin{tabular}{lccl}
\hline Group $(\mathrm{n})$ & $\mathrm{I}(\mathbf{9})$ & $\boldsymbol{\|}(\mathbf{2 2})$ & $\mathbf{p}^{* *}$ \\
\hline Gender M/F & $4 / 5$ & $12 / 10$ & $\mathrm{NS}$ \\
\hline Age (years) & $62.5 \pm 6.9$ & $55.5 \pm 14.3$ & $\mathrm{NS}$ \\
\hline Duration of AF (months) & $9.9 \pm 6.4$ & $4.9 \pm 2.8$ & 0.02 \\
\hline $\begin{array}{l}\text { Highest energy per } \\
\text { shock (Joules) }\end{array}$ & $304.4 \pm 65.4$ & $240.0 \pm 50.3$ & 0.006 \\
\hline Left atrium (mm) & $41.1 \pm 14.5$ & $41.3 \pm 4.7$ & $\mathrm{NS}$ \\
\hline Ejection fraction (\%) & $61.0 \pm 6.3$ & $64.6 \pm 8.5$ & $\mathrm{NS}$ \\
\hline
\end{tabular}

${ }^{*}$ mean $\pm S D .{ }^{* *} p$ value between groups I and II.

spectral turbulence analyses, the values of MEC decreased, for all patients, from the first to the second P-SAECG, but did not decrease from the second to the third one (MEC: $89.2 \pm 7.2 \mathrm{vs}$ $76.5 \pm 8.1$ vs $77.3 \pm 8.7$ for, respectively, first, second and third P-SAECG; first vs. second: $p<0.001$; first vs. third: $p<0.001$; second vs. third: $p=0.34$ ). MET and SET parameters showed similar behavior. Figure 2 shows sequential P-SAECG of Group II acquired immediately after successfully cardioversion and one week later.

\section{Discussion}

In the present study, only subjects with lone AF were enrolled. The term "lone AF" describes this arrhythmia in the absence of demonstrable underlying cardiac disease.

Most studies about electrical atrial remodeling in humans have assessed subjects with and without overt cardiovascular disease $23-25,27$. Although micro structural atrial changes have been observed in lone $\mathrm{AF}^{29}$, gross and ultra-structural anatomic changes due to underlying cardiac disease, such as fibrosis, may persist indefinitely, despite the restoration to sinus rhythm and may be responsible for persistent electrophysiological abnormalities. Therefore, we believe that a lone-AF model would be more representative of the electrophysiological changes process due to the electrical remodeling phenomenon.

Long standing AF is more resistant to cardioversion and more likely to recur ${ }^{30}$. In this scenario, amiodarone has been shown to be more effective than other antiarrhythmic drugs for the maintenance of sinus rhythm ${ }^{31}$. In subjects with AF lasting longer than 3 months, previous use of amiodarone before cardioversion increases the likelihood of restoration to sinus rhythm and prevention of early recurrence of the arrhythmia ${ }^{28}$. Although it may be reasonable to accept that amiodarone promotes changes in the electrophysiological properties during atrial activation, our subjects were assessed under the use of the drug, because this approach is already usual in clinical practice. However, in order to minimize inter-individual variation, we attempted to standardize the amiodarone dose according to body weight.

In previous studies, we demonstrated that spectral turbulence of the P-SAECG identifies subjects at risk of either 
early or frequent recurrence of atrial fibrillation after successful electrical cardioversion and evolution to the permanent form of the arrhythmia ${ }^{6,32}$. Other studies have demonstrated a strong correlation between fractionated endocardial electrograms and AF episodes ${ }^{33,34}$. Fragmented ventricular activation

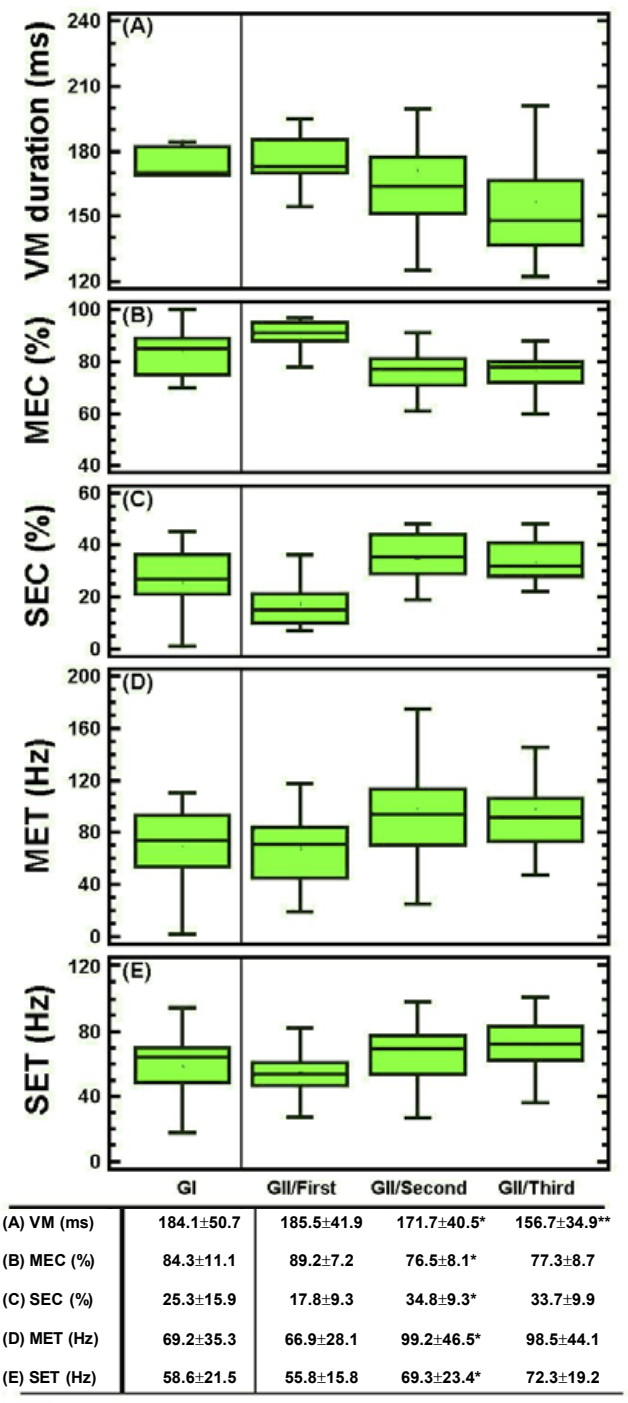

Signal-averaged ECG Follow-up

Figure 1 - From top to bottom, Box-and-Whisker plots of all parameters analyzed in P-wave signal-averaged ECG in Groups I and II, following successful cardioversion of atrial fibrillation to sinus rhythm. P-SAECGs were acquired immediately cardioversion in Groups I and II (/First), and on the seventh (Group II/Second) and the thirtieth (Group II/Third) days. In the table below, statistical comparisons and respective P-SAECG parameters (mean $\pm S D$ ) during follow-up are shown. No differences were found between Groups I and II/First. In Group II, observe a significant reduction in P-wave vector magnitude (VM) duration from the first to the second P-SAECG, paralleled by an increase in spectral turbulence characterized by: i) significant reduction of mean inter-segment spectral correlation (MEC, B), and ii) increases in standard deviation of inter-segment spectral correlation (SEC, C) and mean (MET, E) and standard deviation (SET, F) of the spectral edge track. From the second to the third P-SAECG, a significant difference in VM indicated a continuous shortening of the $P$-wave duration up to the thirtieth day after cardioversion, when follow-up was discontinued. '*' Represents $p<$ 0.05 between the first and the second P-SAECG in Group II. '**' Represents $p<0.05$ between the second and the third P-SAECG in Group II. detected by endocardial electrograms has been already correlated with low-amplitude and high-frequency signals detected on body surface SAECG ${ }^{35,36}$. Thus, it is reasonable to conclude that the spectral turbulence of the surface P-SAECG reflects the presence of potential fragmented intracavitary atrial activation.

In the present study, the P-wave duration progressively abates during the first month after sinus rhythm restoration, which parallels spectral turbulence development. During the first week in sinus rhythm, intense spectral turbulence in atrial electrical activity progressively supervenes, suggesting that early recovery of atrial activation is characterized by nonuniform anisotropic conduction, which remains unchanged until the end of the first month. It is noteworthy that, in our population, spectral turbulence, starting during the first seven days in sinus rhythm, was mostly concentrated in the terminal portion (second half, Figure 2) of the P-wave. The temporal relationship of the high-frequency content detected on the P-wave in the present study is in accordance, at least in part, with the high-frequency fragmentation registered in left atrium during atrial fibrillation by Kalifa et $\mathrm{al}^{33}$.

On the other hand, in the present study, all recurrences occurred in the first week after sinus rhythm restoration. Previous studies using implantable devices for monitoring subjects submitted to electrical cardioversion of atrial fibrillation have demonstrated recurrence rates over 50\%, especially in the first week. Tieleman et al ${ }^{37}$ followed 61 subjects (average of seven months in atrial fibrillation) by transtelephonic monitoring after restoration of sinus rhythm and observed that $22(36 \%)$ recurred as early as five days after successful cardioversion, following progressive and substantial reduction in daily episodes of recurrence. In subjects with mid to long-term atrial fibrillation, the first week that follows conversion to sinus rhythm is characterized by marked vulnerability to arrhythmia recurrence. Vulnerability has been, at least in part, attributed to the reversal of atrial electrical remodeling ${ }^{24,38}$.

P-SAECG vector magnitude duration has been accepted as an index of intra-atrial conduction velocity ${ }^{3,24,39}$ and a marker of atrial fibrillation ${ }^{40}$. Sato et $\mathrm{al}^{39}$ did not observe significant P-wave duration changes from one hour to $24 \mathrm{~h}$ after successful cardioversion of long term atrial fibrillation. Yu et $\mathrm{al}^{3}$ reported that $\mathrm{P}$-wave duration and both inter and intra-atrial conduction times were longer in subjects with atrial fibrillation than controls; however, no reduction in conduction times were observed from immediately after to the following four days post sinus rhythm restoration. Guo et $\mathrm{al}^{41}$ prospectively analyzed 60 patients with persistent AF submitted to successful electrical cardioversion and followed with serial SAECG carried out immediately after and seven 7,30 90 and 180 days after sinus rhythm restoration. The authors found out that in patients who remained in sinus rhythm during follow-up, the filtered P-wave SAECG duration was both shorter and showed faster P-wave shortening than in those who eventually recurred. In contrast to Guo et $\mathrm{al}^{41}$, who observed reduction in P-wave SAECG duration after the first week after sinus rhythm restoration, in our study, the reduction in P-wave SAECG duration was more remarkable during the first week. These differences could be explained by different population arrhythmia profiles. 

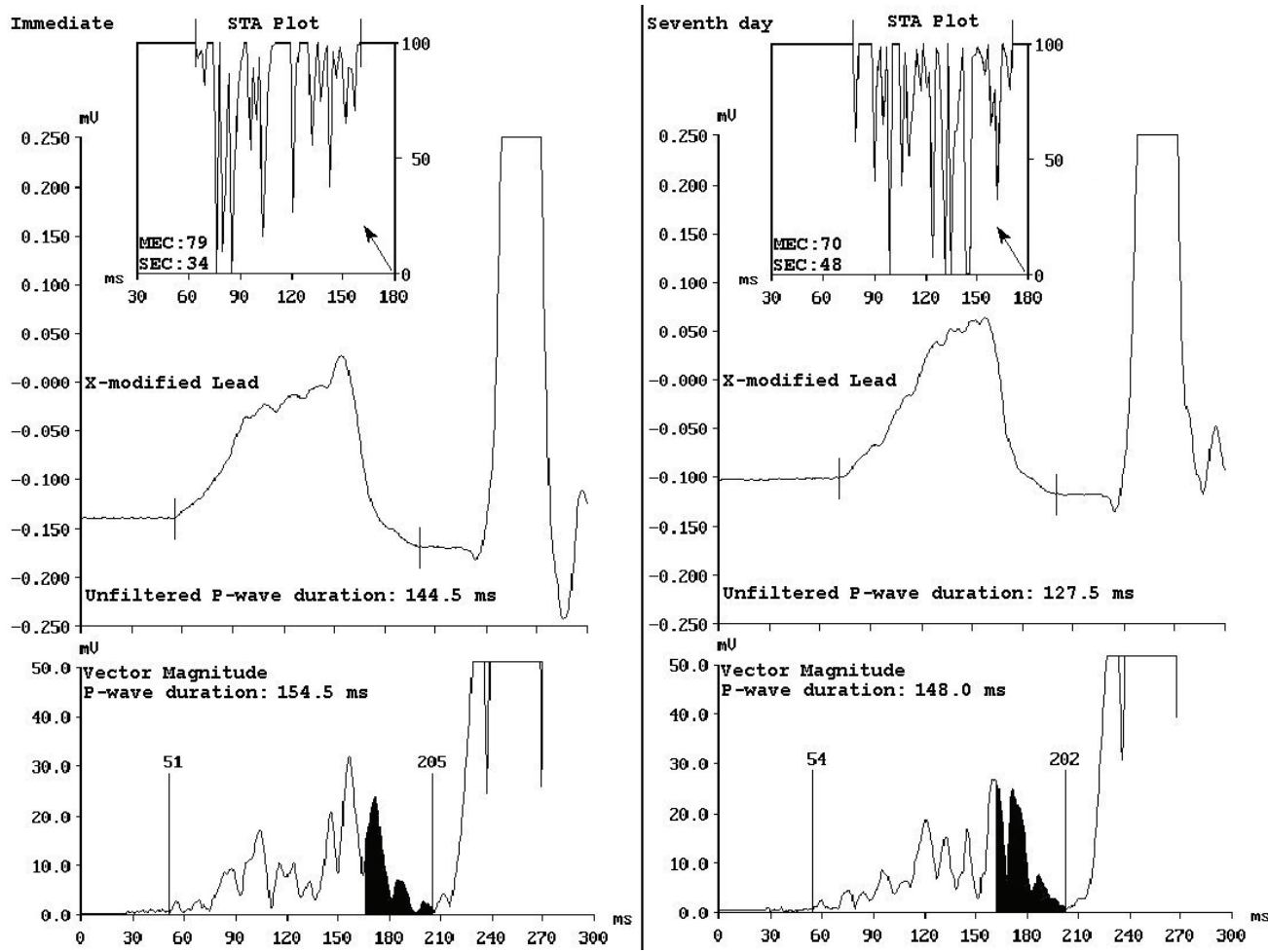

Figure 2 - The frequency and time domain analysis of P-wave signal-averaged ECG (SAECG). On the left-hand-side column, from upper to bottom, spectral turbulence analysis (STA plot), unfiltered X-modified lead and filtered vector magnitude (VM) of the P-wave SAECG immediately after conversion from AF to sinus rhythm. On the right-hand-side column, the SAECG of the same subject seven days after conversion. Observe the shortening of the P-wave (unfiltered: $144.5 \mathrm{~ms}$ vs $127.5 \mathrm{~ms}$ and VM: $154.5 \mathrm{~ms}$ vs $148.0 \mathrm{~ms}$ ) and increasing spectral turbulence (MEC: 79 vs 70 and SEC: 34 vs 48), demonstrating the development of intense high-frequency intra-atrial electrical activity. In the inset (units on the right ordinate are percent values), dark arrow point to the region corresponding the terminal portion of the $P$-wave. Observe increase in spectral turbulence in the second half of the P-wave SAECG recorded on the seventh day as compared to the one recorded immediately after conversion to sinus rhythm. MEC - mean spectral correlation, SEC - standard deviation of spectral correlation.

Whereas in our study, all patients had lone AF and use of anti-arrhythmic drugs was uniform and controlled, in Guo at $\mathrm{al}^{41}$ study most patients had structural cardiomyopathy and were taking different anti-arrhythmic drugs. On the other hand, Chalfoun et $\mathrm{al}^{42}$ followed 60 subjects with persistent AF who underwent successful electrical cardioversion during 30 days. They observed no significant differences in P-wave SAECG duration immediately after cardioversion between those who remained in sinus rhythm (22 patents, 37\%) and those who recurred in the follow-up period. A significant reduction in P-wave SAECG duration between 30 days and immediately after sinus restoration was reported in those who remained in sinus rhythm. However, Chalfoun et al ${ }^{42}$ found that left atrial size at the beginning of the study directly correlated to P-wave SAECG duration immediately after sinus rhythm restoration only among those who were in sinus rhythm after one month of follow-up, suggesting that diverse pathophysiological mechanisms may play relevant roles in AF recurrence. The only predictors of AF recurrence were the presence of left ventricular hypertrophy and previous AF duration. In our study, P-wave duration progressively abated from the immediate sinus rhythm restoration to the $30^{\text {th }}$ day, with remarkable reduction observed in the first week. In Yu et $\mathrm{al}^{3}$ study, although not statistically significant, average $\mathrm{P}$ wave duration did reduce from $144 \mathrm{~ms}$ to $138 \mathrm{~ms}$. Raitt et $\mathrm{al}^{24}$ demonstrated a significant reduction in P-wave duration in subjects with atrial fibrillation lasting more than 12 months when compared to both immediate post-cardioversion and seven days afterwards. Healey et $\mathrm{al}^{43}$ detected a significant P-wave shortening from immediately after to three days post successful reversion of atrial fibrillation. It is noteworthy that in several studies ${ }^{3,24,39}$ no significant lengthening of conduction time in the lateral wall of the left atrium was observed during atrial fibrillation episodes, opposite to inter-atrial conduction time (between the right atrium lateral wall and a distal electrode far in the coronary sinus), which significantly lengthens during atrial fibrillation and progressively reduces after restoration of the sinus rhythm.

Shortening of the atrial refractory period following establishment of atrial fibrillation and further lengthening after sinus rhythm restoration has already been demonstrated in humans as well as in animal models ${ }^{2-5,10-13,23-25}$. However, after long standing atrial fibrillation conversion to sinus rhythm, both lengthening and rate-adaptation of refractory period shows non-uniform time-dependent recovery in the atria, having been observed earlier on the right (hours) than 
on the left atrium (days) thus, predisposing to dispersion of atrial refractoriness. In our study, the analysis of the atrial activation by P-SAECG demonstrated progressive increase in the spectral turbulence after cardioversion, most remarkably observed after seven days of sinus rhythm restoration. As the first week after sinus rhythm recovery coincides with: 1) lengthening of the refractory period and increased conduction velocity, 2) Increased intra-atrial dispersion of refractoriness, and 3) higher rates of atrial fibrillation recurrence, we hypothesized that the increase in the velocity conduction in contrast with the non-homogeneous lengthening of the refractory period provides conditions for non-uniform anisotropism and development of functional barriers in atrial conduction. These changes are the rationale for the progressive increase in spectral turbulence observed in P-SAECG.

\section{Limitations}

In our population, six patients (16\%) were excluded due to unsuccessful electrical cardioversion. The lack of a biphasic electrical cardioverter may have contributed to the present rate of unsuccessful cardioversions. Evidence from intracavitary mapping studies comparing the presence of intra-atrial highfrequency fragmentation regions with signal averaged surface ECG high-frequency content is still lacking. The authors speculate that the sources of high frequency content detected

\section{References}

1. Goette A, Honeycutt C, Langberg JJ. Electrical remodeling in atrial fibrillation: time course and mechanisms. Circulation. 1996; 94: 2968-74.

2. Wijffels MC, Kirchhof CJ, Dorland R, Allessie MA. Atrial fibrillation begets atrial fibrillation: a study in awake chronically instrumented goats. Circulation. 1995; 92: 1954-68.

3. Yu WC, Lee SH, Tai CT, Tsai CF, Hsieh MH, Chen CC, et al. Reversal of atrial electrical remodeling following cardioversion of long-standing atrial fibrillation in man. Cardiovasc Res. 1999; 42: 470-6.

4. Fareh S, Villemaire C, Nattel S. Importance of refractoriness heterogeneity in the enhanced vulnerability to atrial fibrillation induction caused by tachycardia-induced atrial electrical remodeling. Circulation. 1998; 98: 2202-9.

5. Pandozi C, Bianconi L, Villani M, Gentilucci G, Castro A, Altamura G, et al. Electrophysiological characteristics of the human atria after cardioversion of persistent atrial fibrillation. Circulation. 1998; 98: 2860-5.

6. Barbosa PR, de Souza Bomfim A, Barbosa EC, Ginefra P, Boghossian HCS, Destro C, et al: Spectral turbulence analysis of the signal-averaged electrocardiogram of the atrial activation as predictor of recurrence of idiopathic and persistent atrial fibrillation. Int J Cardiol. 2006; 107: 307-16.

7. Suttorp MJ, Kingma JH, Jessurun ER, Lie-A-Huen L, van Hemel NM, Lie KI. The value of class IC antiarrhythmic drugs for acute conversion of paroxismal atrial fibrillation or flutter to sinus rhythm. J Am Coll Cardiol. 1990; 16: 1722-7.

8. Waris E, Kreus KE, Salokannel J. Factors influencing persistence of sinus rhythm after DC shock treatment of atrial fibrillation. Acta Med Scand. 1971; 189: 161-6.

9. Crijns HIGM, van Wijk LM, van Gilst WH, Herre Kingma J, van Gelder IC, Lie KI. Acute cardioversion of atrial fibrillation for sinus rhythm: clinical efficacy of flecainide acetate. Comparison of two regimens. Eur Heart J. 1998;9:634-638.

10. Morillo CA, Klein GJ, Jones DL, Guiraudon CM. Chronic rapid atrial pacing: on P-wave SAECG may be in parallel with fragmented atrial endocardial activity, although invasive studies to support this theory in atria are still needed.

\section{Conclusion}

After restoration of the sinus rhythm in subjects with longstanding atrial fibrillation, there is a progressive shortening of the P duration observed during the first month, especially in the first week, paralleled by the accentuation of high frequency electrical activity on the seventh day, which remains unchanged until the thirtieth day. The intrinsic mechanisms responsible for the present findings await elucidation.

\section{Potential Conflict of Interest}

No potential conflict of interest relevant to this article was reported.

\section{Sources of Funding}

There were no external funding sources for this study.

\section{Study Association}

This article is part of the thesis of doctoral submitted by Eduardo Correa Barbosa, from Universidade do Estado do Rio de Janeiro. structural, functional, and electrophysiological characteristics of a new model of sustained atrial fibrillation. Circulation. 1995; 91: 1588-95.

11. Elvan A, Wylie K, Zipes DP. Pacing-induced chronic atrial fibrillation impairs sinus node function in dogs: electrophysiological remodeling. Circulation. 1996; 94: 2953-60.

12. Nattel S. Atrial electrophysiological remodeling caused by rapid atrial activation: underlying mechanisms and clinical relevance to atrial fibrillation. Cardiovasc Res. 1999; 42: 298-308.

13. Lee SH, Lin FY, Yu WC, Cheng JJ, Kuan P, Hung CR, et al. Regional differences in the recovery course of tachycardia-induced changes of atrial electrophysiological properties. Circulation. 1999; 99: 1255-64.

14. Calò L, Pandozi C, Lamberti F, Riccardi R, Loricchio ML, Castro A, et al. Electrophysiology of atrial fibrillation: evolving insights. Ital Heart J. 2000; 1 : 521-531.

15. Gaspo R, Bosch RF, Talajic M, Nattel S: Functional mechanisms underlying tachycardia-induced sustained atrial fibrillation in a chronic dog model. Circulation. 1997; 96: 4027-35.

16. Papageorgiou P, Monahan K, Boyle NG, Seifert MJ, Beswick P, Zebede J, et al. Site-dependent intra-atrial conduction delay: relationship to initiation of atrial fibrillation. Circulation. 1996; 94: 384-9.

17. Allessie MA, Bonke FI, Schopman FJ. Circus movement in rabbit atrial muscle as a mechanism of tachycardia. II. The role of nonuniform recovery of excitability in the occurrence of unidirectional block, as studied with multiple microelectrodes. Circ Res. 1976; 39: 168-77.

18. Boutjdir M, Le Heuzey JY, Chauvaud S, Guize J, Cousin MT. Inhomogeneity of celular refractoriness in human atrium: factor of arrhythmia? Pacing Clin Elecrophysiol. 1986; 9 (6 Pt 2):1095-100.

19. Moe GK. On the multiple wavelet hyphotesis of AF. Arch Int Pharmacodyn Ter. 1962; 140: 183-8. 
20. Moe GK, Rheinboldt WC, Abildskov JA. A computer model of atrial fibrillation. Am Heart J. 1964; 67: 200-20.

21. Allessie MA, Lammers WJEP, Bonke FIM, Hollen J. Experimental evaluation of Moe's multiple wavelet hyphotesis of AF. In: Zipes DP, Jalife J (eds). Cardiac electrophysiology and arrhythmias. Orlando: Grune and Stratton; 1985. p. 265-75.

22. Todd DM, Fynn SP, Walden AP, Hobbs WJ, Arya S, Garratt CJ. Repetitive 4-week periods of atrial electrical remodeling promote stability of atrial fibrillation: time course of a second factor involved in the self-perpetuation of atrial fibrillation. Circulation. 2004; 109: 1434-9.

23. Hobbs WJ, Fynn S, Todd DM, Wolfson P, Galloway M, Garratt CJ. Reversal of atrial electrical remodeling after cardioversion of persistent atrial fibrillation in humans. Circulation. 2000; 101: 1145-51.

24. Raitt MH, Kusumoto W, Giraud G, McAnulty JH. Reversal of electrical remodeling after cardioversion of persistent atrial fibrillation. J Cardiovasc Electrophysiol. 2004; 15: 507-12.

25. Spurrell P, Kamalvand K, Higson M, Sulke N. Temporal changes in atrial refractoriness following DC cardioversion of persistent atrial fibrillation in man. Europace. 2004;6:229-35.

26. Raitt MH, Kusumoto W, Giraud GD, McAnulty JH. Electrophysiologic predictors of the recurrence of persistent atrial fibrillation within 30 days of cardioversion. Am J Cardiol. 2004; 93: 107-10.

27. Yu WC, Chen SA, Lee SH, Tai CT, Feng AN, Kuo BI, et al. Tachycardia-induced change of atrial refractory period in humans: rate dependency and effects of antiarrhythmic drugs. Circulation. 1998; 97: 2331-7.

28. Manios EG, Mavrakis HE, Kanoupakis EM, Kallergis EM, Dermitzaki DN, Kambouraki DC, et al. Effects of amiodarone and diltiazem on persistent atrial fibrillation conversion and recurrence rates: a randomized controlled study. Cardiovasc Drugs Ther. 2003; 17: 31-9.

29. Frustaci A, Chimenti C, Bellocci F, Morgante E, Russo MA, Maseri A. Histological substrate of atrial biopsies in patients with lone atrial fibrillation. Circulation. 1997; 96: 1180-4.

30. Frick M, Frykman V, Jensen-Urstad M, Ostergren J, Rosenqvist M. Factors predicting success rate and recurrence of atrial fibrillation after first electrical cardioversion in patients with persistent atrial fibrillation. Clin Cardiol. 2001; 24: $238-44$.

31. Chun SH, Sager PT, Stevenson WG, Nademanee K, Middlekauff HR, Singh $\mathrm{BN}$. Long-term efficacy of amiodarone for the maintenance of normal sinus rhythm in patients with refractory atrial fibrillation or flutter. Am J Cardiol. 1995; 76: 47-50

32. Barbosa EC, Barbosa PR, Ginefra P, de Souza Bomfim A, Boghossian SH, da Rocha PJ, et al. The frequency analysis of signal-averaged ECG of P wave as predictor of efficacy of class III antiarrhythmic drugs to maintain sinus rhythm in recurrent idiopathic atrial fibrillation. Ann Noninvasive Electrocardiol. $2001 ; 6: 43-9$

33. Kalifa J, Tanaka K, Zaitsev AV, Warren M, Vaidyanathan R, Auerbach D, et al. Mechanisms of wave fractionation at boundaries of high-frequency excitation in the posterior left atrium of the isolated sheep heart during atrial fibrillation. Circulation. 2006; 113: 626-33.

34. Tanigawa M, Fukatani M, Konoe A, Isomoto S, Kadena M, Hashiba K. Prolonged and fractionated right atrial electrograms during sinus rhythm in patients with paroxysmal atrial fibrillation and sick sinus node syndrome. J Am Coll Cardiol. 1991; 17: 403-8.

35. Aihara N, Ohe T, Shimomura K. Time-domain and frequency-domain analyses of the signal-averaged ECG in patients with sustained ventricular tachyarrhythmia and nonischemic heart diseases. J Electrocardiol. 1994; 27 (Suppl): 194-201.

36. Sasaki Y, Furihata A, Suyama K. Endocardial fragmented electrogram and prediction of ventricular tachycardia by body surface signal-averaged electrocardiographic mapping. Pacing Clin Electrophysiol. 1995; 18: 1479-86.

37. Tieleman RG, van Gelder IC, Crijns HJ, de Kam PJ, Van den Berg MP, Haaksma J, et al. Early recurrences of atrial fibrillation after electrical cardioversion: a result of fibrillation-induced electrical remodeling of the atria? J Am Coll Cardiol. 1998; 31: 167-73.

38. Van Wagoner DR. Electrophysiological remodeling in human atrial fibrillation. Pacing Clin Electrophysiol. 2003; 26 (Pt 3): 1572-5.

39. Sato T, Mitamura H, Kurita Y, Takeshita A, Shinagawa K, Miyoshi S, et al Recovery of electrophysiological parameters after conversion of atrial fibrillation. Int J Cardiol. 2001; 79: 183-9.

40. Moreira JO, Moffa PJ, Uchida AH, Tobias NM, Grupi CJ, Luna Filho B, et al. The signal-averaged electrocardiogram of atrial activation in patients with or without paroxysmal atrial fibrillation. Arq Bras Cardiol. 2006; 87: 564-9.

41. Guo XH, GallagherMM, Poloniecki J, Yi G, Camm AJ. Prognostic significance of serial P wave signal-averaged electrocardiograms following external electrical cardioversion for persistent atrial fibrillation: a prospective study. Pacing Clin Electrophysiol. 2003; 26: 299-304.

42. Chalfoun N, Harnick D, Pe E, Undavia M, Mehta D, Gomes JA. Reverse electrical remodeling of the atria post cardioversion in patients who remain in sinus rhythm assessed by signal averaging of the P-wave. Pacing Clin Electrophysiol. 2007; 30: 502-9.

43. Healey JS, Theoret-Patrick P, Green MS, Lemery R, Birnie D, Tang AS. Reverse atrial electrical remodelling following atrial defibrillation as determined by signal-averaged ECG. Can J Cardiol. 2004; 20: 311-5. 